\title{
Symmetries of the Free Schrödinger Equation in the Non-Commutative Plane ${ }^{\star}$
}

\author{
Carles BATLLE ${ }^{\dagger}$, Joaquim GOMIS ${ }^{\ddagger}$ and Kiyoshi KAMIMURA $§$ \\ † Departament de Matemàtica Aplicada 4 and Institut d'Organització $i$ Control, \\ Universitat Politècnica de Catalunya - BarcelonaTech, EPSEVG, Av. V. Balaguer 1, \\ 08800 Vilanova i la Geltrú, Spain \\ E-mail: carles.batlle@upc.edu \\ ¥Departament d’Estructura i Constituents de la Matèria and Institut de Ciències del Cosmos, \\ Universitat de Barcelona, Diagonal 647, 08028 Barcelona, Spain \\ E-mail:gomis@ecm.ub.es \\ $\S$ Department of Physics, Toho University, Funabashi, Chiba 274-8510, Japan \\ E-mail:kamimura@ph.sci.toho-u.ac.jp
}

Received August 29, 2013, in final form January 29, 2014; Published online February 08, 2014

http://dx.doi.org/10.3842/SIGMA.2014.011

\begin{abstract}
We study all the symmetries of the free Schrödinger equation in the non-commutative plane. These symmetry transformations form an infinite-dimensional Weyl algebra that appears naturally from a two-dimensional Heisenberg algebra generated by Galilean boosts and momenta. These infinite high symmetries could be useful for constructing nonrelativistic interacting higher spin theories. A finite-dimensional subalgebra is given by the Schrödinger algebra which, besides the Galilei generators, contains also the dilatation and the expansion. We consider the quantization of the symmetry generators in both the reduced and extended phase spaces, and discuss the relation between both approaches.
\end{abstract}

Key words: non-commutative plane; Schrödinger equation; Schrödinger symmetries; higher spin symmetries

2010 Mathematics Subject Classification: 81R60; 81S05; 83C65

\section{Introduction and results}

The symmetries of a free massive non-relativistic particle and the associated Schrödinger equation have been investigated. The projective symmetries of the Schrödinger equation induced by the transformation on the coordinates $(t, \vec{x})$ are well known. They form the Schrödinger group $[12,19,20,23]$ that, apart from the Galilei symmetries, contains the dilatation and the expansion. Recently Valenzuela [24] (see also [4]) discussed higher-order symmetries of the free Schrödinger equation. These symmetry transformations form an infinite-dimensional Weyl algebra constructed from the generators of space-translation and the ordinary commuting Galilean boost. The extra symmetries that do not belong to the Schrödinger group correspond to higher spin symmetries. These transformations are not induced by the transformations on the coordinates but they map solutions into solutions of the Schrödinger equation.

In the case of $2+1$ dimensions, the Galilei group admits two central extensions [2, 5, 14, 15, 21], one associated to the exotic non-commuting boost and other appearing in the commutator of the ordinary boost and spatial translations. The non-relativistic particle in the non-commutative plane was introduced in [22] by considering a higher order Galilean invariant Lagrangian for the coordinates $(t, \vec{x})$ of the particle. A first order Lagrangian depending on the coordinates $(t, \vec{x})$

\footnotetext{
${ }^{\star}$ This paper is a contribution to the Special Issue on Deformations of Space-Time and its Symmetries. The
} full collection is available at http://www.emis.de/journals/SIGMA/space-time.html 
and extra coordinates $\vec{v}$ was introduced in [9]. For these Lagrangians there are two possible realizations, one with non-commuting (exotic) boosts, and the other with ordinary commuting boosts [5, 16] (see [15] for a review).

In this paper we study all the infinitesimal Noether symmetries of a massive free particle in the $(2+1)$-dimensional non-commutative plane. The Noether symmetries are constructed from the Heisenberg algebra of commuting boosts $X_{i}$ and the generators of translations $P_{i}$, $\left\{X_{i}, P_{j}\right\}=\delta_{i j}, i, j=1,2$, all of which are constants of motion and are written explicitly in terms of the initial conditions. The algebra of these symmetries is the infinite-dimensional Weyl algebra associated with the Heisenberg algebra. A general element of the Weyl algebra is given by $\mathfrak{G}\left(X_{i}, P_{j}\right)$. The generators given by higher degree polynomials do not form a closed algebra for any finite degree. These infinite symmetries are the non-relativistic counterpart of all the symmetries of the free massless Klein-Gordon equation [10]. There is no known realization of this Weyl algebra for an Schrödinger equation with interaction. These symmetries could be useful to construct a non-relativistic analogue of Vasiliev's higher spin theories [25].

The subset of generators constructed up to quadratic polynomials of $\left(X_{i}, P_{j}\right)$ form a finitedimensional sub-algebra, which in turn contains the 9-dimensional Schrödinger algebra. We study the realization of this algebra in the classical unreduced phase-space, as well as in the reduced one, the later appearing due to the presence of second class constraints. We also study all the symmetries of the free Schrödinger equation in the non-commutative plane. The symmetries are in one to one correspondence with the Noether symmetries of the free particle in the noncommutative plane. This analysis is done in the quantum reduced phase space, as well as in the extended one. In the extended space we impose non-hermitian combinations of the second class constraints. In this case we consider two representations for the physical states, namely a Fock representation [16] and a coordinate representation. We study the Schrödinger subalgebra in detail, and we show the equivalence between the reduced and extended space formulations. We show that, in general, the quadratic (and higher) generators in the extended space contain second order derivatives and hence do not generate point transformations for the coordinates.

The organization of the paper is as follows. In Section 2 we construct all Noether symmetries of the massive particle in the non-commutative plane. Section 3 is devoted to the study of the quantum symmetries of the Schrödinger equation.

\section{Classical symmetries of the non-relativistic particle Lagrangian in the non-commutative plane}

In this section we introduce a first order Lagrangian describing a particle in the non-commutative plane [9], and present the corresponding Hamiltonian formalism. The main result of the section is the construction of all the Noether symmetries of the non-relativistic particle in the non-commutative plane (equations (2.11)-(2.14) and the ensuing discussion). For the sake of completeness, we review the construction of the standard and exotic Galilei algebras and of the Schödinger generators $[3,5,15,16,17]$. We also perform the reduction of the second class constraints of the system for later use in the quantization in the reduced phase space.

The first order Lagrangian of a non-relativistic particle in the non-commutative plane, see for example [9], is given by

$$
\mathcal{L}_{\mathrm{nc}}=m\left(v_{i} \dot{x}_{i}-\frac{v_{i}^{2}}{2}\right)+\frac{\kappa}{2} \epsilon_{i j} v_{i} \dot{v}_{j}, \quad i, j=1,2,
$$

where the overdot means derivative with respect to "time" $t$. This Lagrangian can be obtained using the NLR method [7,6] applied to the exotic Galilei group in $2+1$ dimensions $^{1}$; see [1]

\footnotetext{
${ }^{1}$ Note that this Lagrangian is not dynamically equivalent to the higher order Lagrangian for a non-relativistic
} 
for the case of exotic Newton-Hooke whose flat limit gives (2.1). The coordinates $x_{i}$ 's are the Goldstone bosons of the transverse translations and $v_{i}$ 's are the Goldstone bosons of the broken boost. The $v_{i}$ 's and $\kappa$ are dimensionless.

The Lagrangian (2.1) gives two primary second class constraints

$$
\Pi_{i}=\pi_{i}+\frac{\kappa}{2} \epsilon_{i j} v_{j} \approx 0, \quad V_{i}=p_{i}-m v_{i} \approx 0
$$

where $p_{i}$ and $\pi_{i}$ are the momenta canonically conjugate to $x_{i}$ and $v_{i}$. The constraints $(2.2)$ satisfy relations

$$
\left\{\Pi_{i}, \Pi_{j}\right\}=\kappa \epsilon_{i j}, \quad\left\{V_{i}, V_{j}\right\}=0, \quad\left\{\Pi_{i}, V_{j}\right\}=m \delta_{i j},
$$

and the Dirac Hamiltonian is

$$
H=\frac{p_{i}^{2}}{2 m},
$$

up to quadratic terms in the constraints.

From the canonical pairs $(x, v, p, \pi)$ we can get a new set of canonical pairs $(\tilde{x}, \tilde{v}, \tilde{p}, \tilde{\pi})$ given by

$$
\left(\begin{array}{c}
\tilde{x} \\
\tilde{p} \\
\tilde{v} \\
\tilde{\pi}
\end{array}\right)=\left(\begin{array}{cccc}
1 & -\frac{\kappa}{2 m^{2}} \epsilon & \frac{\kappa}{2 m} \epsilon & -\frac{1}{m} \\
1 & & \\
& -\frac{1}{m} & 1 & \\
\frac{\kappa}{2 m} \epsilon & & 1
\end{array}\right)\left(\begin{array}{l}
x \\
p \\
v \\
\pi
\end{array}\right) .
$$

In terms of the new variables the constraints (2.2) become a canonical pair,

$$
\tilde{v}_{i}=-\frac{1}{m} V_{i} \approx 0, \quad \tilde{\pi}_{i}=\Pi_{i}+\frac{\kappa}{2 m} \epsilon_{i j} V_{j} \approx 0 .
$$

The position and momentum of the particle are expressed as

$$
x_{i}=\tilde{x}_{i}-\frac{\kappa}{2 m^{2}} \epsilon_{i j} \tilde{p}_{j}-\frac{\kappa}{2 m} \epsilon_{i j} \tilde{v}_{j}+\frac{1}{m} \tilde{\pi}_{i}, \quad p_{i}=\tilde{p}_{i},
$$

and the Dirac Hamiltonian (2.3) is written as

$$
H=\frac{1}{2 m} \tilde{p}_{i}^{2} .
$$

The phase space is a direct product of two spaces. One is spanned by $(\tilde{v}, \tilde{\pi})$ with the constraints $(2.5)$

$$
\tilde{v}_{i} \approx 0, \quad \tilde{\pi}_{i} \approx 0
$$

and thus classically trivial. The other one is spanned by $(\tilde{x}, \tilde{p})$ with the Hamiltonian $(2.7)$. It is a system of a free non-relativistic particle in $2+1$ dimensions but with the coordinates $\tilde{x}_{i}$. In the classical reduced phase space defined by the second class constraints (2.8) the coordinates $x_{i}$ become non-commutative (see also Subsection 2.1),

$$
\left\{x_{i}, x_{j}\right\}^{*}=\left\{\tilde{x}_{i}-\frac{\kappa}{2 m^{2}} \epsilon_{i k} \tilde{p}_{k}, \tilde{x}_{j}-\frac{\kappa}{2 m^{2}} \epsilon_{j \ell} \tilde{p}_{\ell}\right\}=\frac{\kappa}{m^{2}} \epsilon_{i j} .
$$

particle proposed in [22]. It can be obtained from (2.1) using the inverse Higgs mechanism [18]. 
If we consider a point transformation $(x, v) \rightarrow(y, u)$

$$
y_{i}=x_{i}+\frac{\kappa}{2 m} \epsilon_{i j} v_{j}, \quad u_{i}=v_{i},
$$

in the Lagrangian (2.1) it becomes

$$
\mathcal{L}=m\left(u_{i} \dot{y}_{i}-\frac{u_{i}^{2}}{2}\right),
$$

which is the Lagrangian of a free non-relativistic particle with the commutative coordinates $y_{i}$. Although it has a form of free particle we keep $x_{i}$ as the "position coordinates" of this system. Local interactions would be introduced at the position $x_{i}$ rather than $y_{i}$. The coordinates $y_{i}$ in (2.10) are identified with the commuting coordinates $\tilde{x}_{i}$ in (2.6), while $x_{i}$ are non-commutative as in (2.9).

All the Noether symmetries are generated by constants of motion which are arbitrary functions $\mathfrak{G}\left(X_{i}, P_{j}\right)$ of

$$
X_{i}=\tilde{x}_{i}(0)=\tilde{x}_{i}(t)-\frac{t}{m} \tilde{p}_{i}(t) \quad \text { and } \quad P_{i}=\tilde{p}_{i}(0)=\tilde{p}_{i}(t),
$$

verifying

$$
\left\{P_{i}, P_{j}\right\}=0, \quad\left\{X_{i}, P_{j}\right\}=\delta_{i j}, \quad\left\{X_{i}, X_{j}\right\}=0 .
$$

The Lagrangian (2.1) is quasi-invariant under the transformation generated by $\mathfrak{G}\left(X_{i}, P_{j}\right)$. The canonical variations of $(x, v)$ are

$$
\delta x_{i}=\frac{\partial \mathfrak{G}}{\partial p_{i}}=\frac{\partial \mathfrak{G}}{\partial P_{i}}-\frac{t}{m} \frac{\partial \mathfrak{G}}{\partial X_{i}}+\frac{\kappa}{2 m^{2}} \epsilon_{i j} \frac{\partial \mathfrak{G}}{\partial X_{j}}, \quad \delta v_{i}=\frac{\partial \mathfrak{G}}{\partial \pi_{i}}=-\frac{1}{m} \frac{\partial \mathfrak{G}}{\partial X_{i}} .
$$

When computing the variation of the Lagrangian (2.1) under (2.12), the $\left(p_{i}, \pi_{i}\right)$ are replaced, using the definition of momenta (2.2), by

$$
p_{i} \rightarrow m v_{i}, \quad \pi_{i} \rightarrow-\frac{\kappa}{2} \epsilon_{i j} v_{j}, \quad X_{i} \rightarrow x_{i}-t v_{i}+\frac{\kappa}{2 m} \epsilon_{i j} v_{j} .
$$

It follows that the variation of the Lagrangian becomes a total derivative,

$$
\begin{aligned}
\delta \mathcal{L}_{\mathrm{nc}}=\frac{\mathrm{d}}{\mathrm{d} \tau} & \mathfrak{F}(x, v, t), \\
\mathfrak{F}(x, v, t) & =\left[p_{i} \delta x_{i}+\pi_{i} \delta v_{i}-\mathfrak{G}\right]_{p_{i}=m v_{i}, \pi_{i}=-\frac{\kappa}{2} \epsilon_{i j} v_{j}} \\
\quad & {\left[m v_{i}\left(\frac{\partial \mathfrak{G}}{\partial P_{i}}-\frac{t}{m} \frac{\partial \mathfrak{G}}{\partial X_{i}}\right)-\mathfrak{G}\right]_{p_{i}=m v_{i}, \pi_{i}=-\frac{\kappa}{2} \epsilon_{i j} v_{j}} . }
\end{aligned}
$$

All these Noether symmetries generate an infinite-dimensional Weyl algebra. The Weyl algebra, denoted by $\left[\mathfrak{h}_{2}^{*}\right]$, can be defined [24] as the one generated by (the Weyl ordered) polynomials of the Heisenberg algebra generators, $\left(X_{i}, P_{i}\right)$, that we indicate by $\mathfrak{G}\left(X_{i}, P_{j}\right)$. [ [ $\left.\mathfrak{h}_{2}^{*}\right]$ is the infinitedimensional algebra of a particle in the non-commutative plane. These infinite symmetries are the non-relativistic counterpart of the complete set of symmetries of the free massless KleinGordon equation [10]. The existence of a realization of this Weyl algebra for an interacting Schrödinger equation is an interesting open question.

There are finite-dimensional subalgebras of the higher spin algebra whose generators are constructed from the product of generators $X_{i}, P_{j}$ up to second order:

$$
\mathfrak{h}_{2} \subset \text { Galilei } \subset \operatorname{Sch}(2) \subset \mathfrak{h}_{2} \oplus \mathfrak{s p}(4) \subset\left[\mathfrak{h}_{2}^{*}\right] .
$$

$\operatorname{Sch}(2)$ is the Schrödinger algebra ${ }^{2}$ in $2 \mathrm{D}$, whose generators are those of the Galilean algebra $X_{i}$, $P_{i}, H, J$, together with the dilatation, $D$, and the expansion, $C$.

\footnotetext{
${ }^{2} \mathrm{~A}$ field theory realization of this algebra was given in [14].
} 
Let us restrict now to Galilean and Schrödinger symmetries. We start by considering the Galilean symmetries of (2.1). The action is invariant under translations,

$$
x_{i}^{\prime}=x_{i}+\alpha_{i}, \quad v_{i}^{\prime}=v_{i},
$$

boosts,

$$
x_{i}^{\prime}=x_{i}-\beta_{i} t, \quad v_{i}^{\prime}=v_{i}-\beta_{i},
$$

rotations,

$$
x_{i}^{\prime}=x_{i} \cos \varphi+\epsilon_{i j} x_{j} \sin \varphi, \quad v_{i}^{\prime}=v_{i} \cos \varphi+\epsilon_{i j} v_{j} \sin \varphi,
$$

and time translations

$$
t^{\prime}=t-\gamma
$$

The corresponding Noether charges of translations and boosts are given by

$$
P_{i}=p_{i}, \quad K_{i}=m x_{i}-p_{i} t-\pi_{i}+\frac{\kappa}{2} \epsilon_{i j} v_{j}=m X_{i}+\frac{\kappa}{2 m} \epsilon_{i j} P_{j},
$$

while the angular momentum is

$$
J=\epsilon_{i j}\left(x_{i} p_{j}+v_{i} \pi_{j}\right)=\epsilon_{i j}\left(X_{i} P_{j}+\tilde{v}_{i} \tilde{\pi}_{j}\right) .
$$

Together with the total Hamiltonian (2.3), they generate the exotic Galilei algebra [2, 5, 14, $15,21]$

$$
\begin{aligned}
& \{H, J\}=0, \quad\left\{H, K_{i}\right\}=-P_{i}, \quad\left\{H, P_{i}\right\}=0, \quad\left\{J, P_{i}\right\}=\epsilon_{i j} P_{j}, \\
& \left\{J, K_{i}\right\}=\epsilon_{i j} K_{j}, \quad\left\{K_{i}, P_{j}\right\}=m \delta_{i j}, \quad\left\{K_{i}, K_{j}\right\}=-\kappa \epsilon_{i j}, \quad\left\{P_{i}, P_{j}\right\}=0 .
\end{aligned}
$$

From this, it may seem that the Lagrangian (2.1) gives a phase space realization of the $(2+1)$ dimensional Galilei group with two central charges $m, \kappa$. However, one of the central charges is trivial since, if we modify the generator of the boost as in $[5,13]$,

$$
\tilde{K}_{i}=K_{i}-\frac{\kappa}{2 m} \epsilon_{i j} P_{j}=m X_{i}=m x_{i}-\pi_{i}+\frac{1}{2} \kappa \epsilon_{i j} v_{j}-\frac{\kappa}{2 m} \epsilon_{i j} p_{j}-p_{i} t,
$$

one gets that $(H, P, \tilde{K}, J)$ verifies the standard Galilean algebra without $\kappa .^{3}$ Physically, the result of changing the boost generators is a shift in the parameter of the translations

$$
\alpha_{i} \rightarrow \alpha_{i}+\frac{\kappa}{2 m} \epsilon_{i j} \beta_{j}
$$

Note that the modified boost generators $\tilde{K}_{i}$ are proportional to the coordinates at $t=0$, $X_{i}=\tilde{x}^{i}(0)$, that verify $\left\{X_{i}, X_{j}\right\}=0$, and we have a realization with only one non-trivial central charge associated to the mass of the particle ${ }^{4}$.

The Schrödinger generators are those of the Galilean algebra $X_{i}, P_{i}, H, J$, and the dilatation, $D$, and the expansion, $C$, given by

$$
D=X_{i} P_{i}=x_{i} p_{i}-\frac{t}{m} p_{i}^{2}-\frac{1}{m} \pi_{i} p_{i}+\frac{\kappa}{2 m} \epsilon_{i j} p_{i} v_{j},
$$

\footnotetext{
${ }^{3}$ If we introduce an interaction with a background field this statement is no longer true, since it depends on which coordinates (commutative or non-commutative) are used to define the interaction; see [8, 9, 15, 17]. Notice however that the background field will break, in general, part of the symmetries of the Galilei group.

${ }^{4}$ Note however that $\delta_{K_{i}} L=\delta_{\tilde{K}_{i}} L=\frac{\mathrm{d}}{\mathrm{d} t}\left(-m x_{i}-\frac{\kappa}{2} \epsilon_{i j} v_{j}\right) \beta_{i}$, where $\beta_{i}$ is boost parameter.
} 


$$
\begin{aligned}
C= & m X_{i} X_{i}=m x_{i}^{2}+\frac{1}{m} t^{2} p_{i}^{2}+\frac{1}{m} \pi_{i}^{2}+\frac{\kappa^{2}}{4 m} v_{i}^{2}+\frac{\kappa^{2}}{4 m^{3}} p_{i}^{2}-2 t x_{i} p_{i}-2 x_{i} \pi_{i}+\kappa \epsilon_{i j} x_{i} v_{j} \\
& -\frac{\kappa}{m} \epsilon_{i j} x_{i} p_{j}+\frac{2}{m} t p_{i} \pi_{i}-\frac{\kappa}{m} t \epsilon_{i j} p_{i} v_{j}-\frac{\kappa}{m} \epsilon_{i j} \pi_{i} v_{j}+\frac{\kappa}{m^{2}} \epsilon_{i j} \pi_{i} p_{j}-\frac{\kappa^{2}}{2 m^{2}} v_{i} p_{i} .
\end{aligned}
$$

In the same spirit, we also redefine the generator of rotations as

$$
J=\epsilon_{i j} X_{i} P_{j}=\epsilon_{i j} x_{i} p_{j}-\frac{\kappa}{2 m^{2}} p_{i}^{2}+\frac{\kappa}{2 m} v_{i} p_{i}+\frac{1}{m} \epsilon_{i j} p_{i} \pi_{j},
$$

which, up to square of constraints, coincides with (2.15).

The new, non-zero Poisson brackets are

$$
\begin{array}{lrl}
\{D, C\}=-2 C, \quad\{D, H\}=2 H, & \{H, C\}=-2 D \\
\left\{D, P_{i}\right\}=P_{i}, \quad\left\{D, X_{i}\right\}=-X_{i}, \quad\left\{C, P_{i}\right\}=2 m X_{i} .
\end{array}
$$

The transformations of the coordinates $x_{i}, v_{i}$ under dilatation and expansion are obtained from (2.12) as

$$
\begin{aligned}
& \delta_{D} x_{i}=\frac{\alpha}{m}\left(m x_{i}-2 m t v_{i}+\kappa \epsilon_{i j} v_{j}\right), \quad \delta_{D} v_{i}=-\alpha v_{i}, \\
& \delta_{C} x_{i}=\frac{\lambda}{m}\left(2 m t^{2} v_{i}-2 m t x_{i}+\kappa \epsilon_{i j} x_{j}-2 \kappa t \epsilon_{i j} v_{j}-\frac{\kappa^{2}}{2 m} v_{i}\right), \\
& \delta_{C} v_{i}=\frac{\lambda}{m}\left(-2 m x_{i}+2 m t v_{i}-\kappa \epsilon_{i j} v_{j}\right),
\end{aligned}
$$

where $\alpha$ and $\lambda$ are the corresponding infinitesimal parameters.

\subsection{Reduction of second class constraints}

The classical symmetry algebra is also realized in the reduced phase space defined by the second class constraints $\Pi_{i}=V_{i}=0$. The Dirac bracket is

$$
\{A, B\}^{*}=\{A, B\}+\left\{A, \Pi_{i}\right\} \frac{1}{m}\left\{V_{i}, B\right\}-\left\{A, V_{i}\right\} \frac{1}{m}\left\{\Pi_{i}, B\right\}-\left\{A, V_{i}\right\} \frac{\kappa \epsilon_{i j}}{m^{2}}\left\{V_{j}, B\right\}
$$

and yields

$$
\left\{x_{i}, x_{j}\right\}^{*}=\frac{\kappa}{m^{2}} \epsilon_{i j}, \quad\left\{x_{i}, p_{j}\right\}^{*}=\delta_{i j}, \quad\left\{p_{i}, p_{j}\right\}^{*}=0 .
$$

In this space, the symmetry transformations are generated using the Dirac bracket and the reduced generators, which can be obtained by substituting $v_{i}=p_{i} / m, \pi_{i}=-\kappa /(2 m) \epsilon_{i j} p_{j}$ into the standard ones.

The infinite Weyl symmetries are generated by

$$
\mathfrak{G}^{(R)}\left(x_{i}, p_{j}\right)=\left.\mathfrak{G}\left(X_{i}, P_{j}\right)\right|_{v_{i}=p_{i} / m, \pi_{i}=-\kappa /(2 m) \epsilon_{i j} p_{j}} .
$$

In particular the Schrödinger generators are given by [3]

$$
\begin{aligned}
& P_{i}^{(R)}=p_{i}, \\
& K_{i}^{(R)}=m x_{i}-t p_{i}+\frac{\kappa}{m} \epsilon_{i j} p_{j} \quad \text { (exotic Galilei), } \\
& \tilde{K}_{i}^{(R)}=K_{i}^{(R)}-\frac{\kappa}{2 m} \epsilon_{i j} P_{j}^{(R)}=m x_{i}-t p_{i}+\frac{\kappa}{2 m} \epsilon_{i j} p_{j} \quad \text { (standard Galilei), } \\
& H^{(R)}=\frac{1}{2 m} p_{i}^{2},
\end{aligned}
$$




$$
\begin{aligned}
J^{(R)} & =\epsilon_{i j} x_{i} p_{j}+\frac{\kappa}{2 m^{2}} p_{i}^{2}, \\
D^{(R)} & =p_{i} x_{i}-\frac{1}{m} t p_{i}^{2}, \\
C^{(R)} & =m x_{i}^{2}+\frac{1}{m} t^{2} p_{i}^{2}+\frac{\kappa^{2}}{4 m^{3}} p_{i}^{2}-2 t x_{i} p_{i}+\frac{\kappa}{m} \epsilon_{i j} x_{i} p_{j} .
\end{aligned}
$$

They generate the Schrödinger algebra with the Dirac bracket, since $\tilde{K}_{i}^{(R)}, P_{i}^{(R)}$ generate a Heisenberg algebra:

$$
\left\{\tilde{K}_{i}^{(R)}, P_{j}^{(R)}\right\}^{*}=m \delta_{i j}, \quad\left\{P_{i}^{(R)}, P_{j}^{(R)}\right\}^{*}=0, \quad \text { and } \quad\left\{\tilde{K}_{i}^{(R)}, \tilde{K}_{j}^{(R)}\right\}^{*}=0 .
$$

Symmetry transformations are generated either using the Poisson brackets in the original phase space or using the Dirac brackets with the reduced generators, (2.17)-(2.23). For example the "exotic Galilei" generators $K_{i}$ satisfy

$$
\left\{K_{i}, K_{j}\right\}=\left\{K_{i}^{(R)}, K_{j}^{(R)}\right\}^{*}=-\kappa \epsilon_{i j}
$$

and generate "standard(covariant) Galilei" transformation of $\left(x_{i}, p_{i}\right)$ as

$$
\begin{aligned}
& \delta x_{i}=\left\{x_{i}, \beta \cdot K\right\}=\left\{x_{i}, \beta \cdot K^{(R)}\right\}^{*}=-t \beta_{i}, \\
& \delta p_{i}=\left\{p_{i}, \beta \cdot K\right\}=\left\{p_{i}, \beta \cdot K^{(R)}\right\}^{*}=-m \beta_{i} .
\end{aligned}
$$

The "standard Galilei" generators $\tilde{K}_{i}$ satisfy

$$
\left\{\tilde{K}_{i}, \tilde{K}_{j}\right\}=\left\{\tilde{K}_{i}^{(R)}, \tilde{K}_{j}^{(R)}\right\}^{*}=0
$$

and generate "exotic Galilei" (non-covariant) transformations of $x_{i}, p_{i}$,

$$
\begin{aligned}
& \delta x_{i}=\left\{x_{i}, \beta \cdot \tilde{K}\right\}=\left\{x_{i}, \beta \cdot \tilde{K}^{(R)}\right\}^{*}=-t \beta_{i}+\frac{\kappa}{2 m} \epsilon_{i j} \beta_{j}, \\
& \delta p_{i}=\left\{p_{i}, \beta \cdot \tilde{K}\right\}=\left\{p_{i}, \beta \cdot \tilde{K}^{(R)}\right\}^{*}=-m \beta_{i} .
\end{aligned}
$$

\section{Quantum symmetries of free Schrödinger equation in the non-commutative plane}

In this section we will study the quantization of the model at the level of the Schrödinger equation and their symmetries. We will quantize it in two approaches, one in the reduced phase space and the other in the extended phase space.

\subsection{Quantization in the reduced phase space}

In the classical theory, $x_{i}$ has a nonzero Dirac bracket $\left\{x_{i}, x_{j}\right\}^{*}$ as in (2.16) in the reduced phase space. Since Dirac brackets are replaced by commutators in the canonical quantization, one cannot have a $x_{i}$-coordinate representation of quantum states ${ }^{5}$. To discuss symmetries of Schrödinger equations we introduce new coordinates

$$
y_{i} \equiv x_{i}+\frac{\kappa}{2 m^{2}} \epsilon_{i j} p_{j}, \quad q_{i}=p_{i}
$$

\footnotetext{
${ }^{5}$ Since $p_{i}$ 's are commuting the momentum representation is possible [9].
} 
such that

$$
\left\{y_{i}, y_{j}\right\}^{*}=0, \quad\left\{y_{i}, q_{j}\right\}^{*}=\delta_{i j}, \quad\left\{q_{i}, q_{j}\right\}^{*}=0
$$

The coordinate $y_{i}$ is the one introduced in (2.10) and $q_{i}$ is its conjugate. In these coordinates, the Schrödinger equation $\left(\mathrm{i} \partial_{t}-H\right)|\Psi(t)\rangle=0$ takes the form corresponding to a free particle for the wave function

$$
\Psi(y, t)=\langle y \mid \Psi(t)\rangle, \quad \hat{y}_{i}|y\rangle=y_{i}|y\rangle, \quad\left\langle y \mid y^{\prime}\right\rangle=\delta^{2}\left(y-y^{\prime}\right),
$$

i.e.

$$
\left(\mathrm{i} \partial_{t}-\frac{1}{2 m}\left(-\mathrm{i} \partial_{y}\right)^{2}\right) \Psi(y, t)=0
$$

and the inner product is

$$
\langle\Psi \mid \Psi\rangle=\int \mathrm{d} y \overline{\Psi(y, t)} \Psi(y, t) .
$$

Note that $y_{i}$ are not covariant under exotic Galilei transformation generated by $K_{i}$

$$
\delta y_{i}=\left\{y_{i}, \beta \cdot K\right\}=\left\{y_{i}, \beta \cdot K^{(R)}\right\}^{*}=-\beta_{i} t-\frac{\kappa}{2 m} \epsilon_{i j} \beta_{j},
$$

but covariant under the Galilei transformation generated by $\tilde{K}_{i}$

$$
\delta y_{i}=\left\{y_{i}, \beta \cdot \tilde{K}\right\}=\left\{y_{i}, \beta \cdot \tilde{K}^{(R)}\right\}^{*}=-\beta_{i} t .
$$

The position operators, covariant under $K_{i}$, are

$$
\hat{x}_{i}=y_{i}-\frac{\kappa}{2 m^{2}} \epsilon_{i j}\left(-\mathrm{i} \partial_{y_{j}}\right) .
$$

They are hermitian since $\hat{y}_{i}=y_{i}, \hat{q}_{i}=-\mathrm{i} \partial_{y_{i}}$, with appropriate boundary conditions on $\Psi(y, t)$, are hermitian.

Although in the free theory we are able to work with both the commutative $\hat{y}_{i}=y_{i}$ and the non-commutative $\hat{x}_{i}=y_{i}-\frac{\kappa}{2 m^{2}} \epsilon_{i j}\left(-\mathrm{i} \partial_{y_{j}}\right)$ position operators, this may not be the case in an interacting theory. For example, if we consider an interaction with a background electromagnetic field, which introduces couplings with a source at position $x_{i}$, the non-commutative coordinates are naturally selected (see, for example, $[8,9,15,17])$. If we denote generically by $\mathfrak{G}^{(R)}(t, x, p)=$ $\left.\mathfrak{G}(X, P)\right|_{\Pi=V=0}$ the generators of the Weyl algebra in the reduced classical space, the generators in this quantization are given by

$$
\hat{\mathfrak{G}}_{i}^{(1)}(t, y, \hat{q})=\left.\mathfrak{G}_{i}^{(R)}\right|_{x_{j}=y_{j}-\frac{\kappa}{2 m^{2}} \epsilon_{j l} \hat{q}_{l}, p_{j}=\hat{q}_{j}}=\mathfrak{G}_{i}\left(y-\frac{t}{m} \hat{q}, \hat{q}\right),
$$

with $\hat{q}_{i}=-\mathrm{i} \partial / \partial y_{i}$ and with the appropriate dealing of operator ordering.

The knowledge of all the symmetries of the Schrödinger equation in terms of the coordinates $y^{i}, \hat{y}_{i}$ is the non-commutative analog in $2+1$ dimensions of the high spin symmetries of the relativistic massless Klein Gordon equation [10]. The Vasiliev [25] non-linear theory has these high spin symmetries. In this sense these high spin-nonrelativistic symmetries could be useful in order to construct a non-relativistic Vasiliev theory [24].

We consider next in detail the Schrödinger generators, given by

$$
\hat{P}_{i}^{(1)}=\hat{q}_{i}=-\mathrm{i} \frac{\partial}{\partial y_{i}}
$$




$$
\begin{aligned}
& \hat{\tilde{K}}_{i}^{(1)}=m y_{i}-t \hat{q}_{i}=m y_{i}+\mathrm{i} t \frac{\partial}{\partial y_{i}}, \\
& \hat{H}^{(1)}=\frac{1}{2 m} \hat{q}_{i}^{2}=-\frac{1}{2 m} \frac{\partial^{2}}{\partial y_{i}{ }^{2}}, \\
& \hat{J}^{(1)}=\epsilon_{i j} y_{i} \hat{q}_{j}=-\mathrm{i} \epsilon_{i j} y_{i} \frac{\partial}{\partial y_{j}}, \\
& \hat{D}^{(1)}=y_{i} \hat{q}_{i}-\mathrm{i}-\frac{1}{m} t \hat{q}_{i}^{2}=-\mathrm{i} y_{i} \frac{\partial}{\partial y_{i}}+\frac{1}{m} t \frac{\partial^{2}}{\partial y_{i}{ }^{2}}-\mathrm{i}, \\
& \hat{C}^{(1)}=m y_{i}^{2}-2 t y_{i} \hat{q}_{i}+2 \mathrm{i} t+\frac{1}{m} t^{2} \hat{q}_{i}^{2}=m y_{i}^{2}+2 \mathrm{i} t y_{i} \frac{\partial}{\partial y_{i}}-\frac{1}{m} t^{2} \frac{\partial^{2}}{\partial y_{i}{ }^{2}}+2 \mathrm{i} t,
\end{aligned}
$$

where a Weyl ordering has been used for $\hat{D}^{(1)}$ and $\hat{C}^{(1)}$. These generators are hermitian operators when acting on the wave functions $\Psi(t, y)$. Furthermore, they obey the abstract quantum Schrödinger algebra off shell, with non-zero commutators given by

$$
\begin{aligned}
& {\left[\hat{\tilde{K}}_{i}, \hat{P}_{j}\right]=\mathrm{i} m \delta_{i j}, \quad\left[\hat{J}, \hat{P}_{i}\right]=\mathrm{i} \epsilon_{i j} \hat{P}_{j}, \quad\left[\hat{J}, \hat{\tilde{K}}_{i}\right]=\mathrm{i} \epsilon_{i j} \hat{\tilde{K}}_{j}, \quad\left[\hat{H}, \hat{\tilde{K}}_{i}\right]=-\mathrm{i} \hat{P}_{i},} \\
& {[\hat{D}, \hat{H}]=2 \mathrm{i} \hat{H}, \quad\left[\hat{D}, \hat{P}_{i}\right]=\mathrm{i} \hat{P}_{i}, \quad\left[\hat{D}, \hat{\tilde{K}}_{i}\right]=-\mathrm{i} \hat{\tilde{K}}_{i},} \\
& {[\hat{D}, \hat{C}]=-2 \mathrm{i} \hat{C}, \quad[\hat{H}, \hat{C}]=-2 \mathrm{i} \hat{D}, \quad\left[\hat{C}, \hat{P}_{i}\right]=2 \mathrm{i} \hat{\tilde{K}}_{i} .}
\end{aligned}
$$

Using these, together with

$$
\left[\mathrm{i} \partial_{t}, \hat{\tilde{K}}_{i}^{(1)}\right]=-\mathrm{i} \hat{P}_{i}^{(1)}, \quad\left[\mathrm{i} \partial_{t}, \hat{D}^{(1)}\right]=-2 \mathrm{i} \hat{H}^{(1)}, \quad\left[\mathrm{i} \partial_{t}, \hat{C}^{(1)}\right]=-2 \mathrm{i} \hat{D}^{(1)},
$$

one can show that

$$
\left[\mathrm{i} \partial_{t}-\hat{H}^{(1)}, \hat{\mathfrak{G}}_{i}^{(1)}\right]=0
$$

for all the generators $\hat{\mathfrak{G}}_{i}^{(1)}$, which proves the invariance of the Schrödinger equation under the Schrödinger transformations in this reduced space quantization.

Under a general Weyl transformation, the wave functions transform as

$$
\Psi^{\prime}(y, t)=e^{\mathrm{i} \alpha_{i} \hat{\mathfrak{E}}_{i}^{(1)}\left(t, y,\left(-\mathrm{i} \partial_{y}\right)\right)} \Psi(y, t)
$$

where the $\alpha_{i}$ are the parameters of the transformations. In particular, for the on-shell Schrödinger transformations one has

$$
\Psi^{\prime}(y, t)=e^{A+i B} \Psi\left(y^{\prime}, t^{\prime}\right),
$$

where the coordinate transformations of $(y, t)$ are those of the $N=1$ conformal Galilean transformation, and the multiplicative factor is $e^{A+i B}$, with $A$ and $B$ real functions of the coordinates and of the parameters of the transformation given by (see, for instance, $[11,23]$ )

1) $H$ (time translation),

$$
t^{\prime}=t+a, \quad y^{\prime}=y, \quad A=B=0,
$$

2) $D$ (dilatation)

$$
t^{\prime}=e^{\lambda} t, \quad y^{\prime}=e^{\frac{\lambda}{2}} y, \quad A=\frac{\lambda}{2}, \quad B=0,
$$


3) $C$ (expansion),

$$
t^{\prime}=\frac{t}{1-\kappa t}, \quad y_{i}^{\prime}=\frac{y_{i}}{1-\kappa t}, \quad e^{A}=\frac{1}{(1-\kappa t)}, \quad B=-\frac{\kappa m y^{2}}{2(1-\kappa t)},
$$

4) (spatial translations and boost)

$$
t^{\prime}=t, \quad y_{i}^{\prime}=y_{i}+\left(\beta^{0}+t \frac{\beta^{1}}{m}\right)_{i}, \quad A=0, \quad B=-m\left(y_{i}+\frac{1}{2}\left(\beta_{i}^{0}+t \frac{\beta_{i}^{1}}{m}\right)\right) \frac{\beta_{i}^{1}}{m},
$$

with $\left[\beta_{i}^{0}\right]=L,\left[\beta_{i}^{1}\right]=L^{-1}$.

The difference with respect to the transformation of the ordinary Schrödinger equation is that in the non-commutative case the coordinates that are transformed by conformal Galilean transformations are the canonical ones $y_{i}$, and not the physical position of the particle, $x_{i}$.

The invariance of the solutions of the Schrödinger equation under a general element of the Weyl algebra can be proved using the invariance under the generators of the Heisenberg algebra and the commutators (3.10).

\subsection{Quantization in the extended phase space}

\subsubsection{Fock representation}

In order to quantize the model in the extended phase space the second class constraints (2.2) are imposed as physical state conditions by taking their non-hermitian combinations as in [1]. We first consider the canonical transformation (2.4) that separates the second class constraints as new coordinates. It is realized at quantum level as a unitary transformation

$$
\tilde{q}=U^{\dagger} q U, \quad U=e^{\frac{i}{m} p_{i}\left(\pi_{i}-\frac{\kappa}{2} \epsilon_{i j} v_{j}\right)} .
$$

For example,

$$
\tilde{x}_{i}=U^{\dagger} x_{i} U=x_{i}-\frac{1}{m}\left(\pi_{i}-\frac{\kappa}{2} \epsilon_{i j} v_{j}\right)+\frac{1}{2} \frac{\kappa}{m} \epsilon_{i j}\left(-\frac{p_{j}}{m}\right) .
$$

It is useful to introduce the complex combinations of the phase space variables $\tilde{\pi}_{ \pm}=\tilde{\pi}_{1} \pm \mathrm{i} \tilde{\pi}_{2}$ and $\tilde{v}_{ \pm}=\tilde{v}_{1} \pm \mathrm{i} \tilde{v}_{2}$, which allow us to introduce two pairs of annihilation and creation operators

$$
\tilde{a}_{ \pm}=\frac{\mathrm{i}}{\sqrt{2 \kappa}}\left(\tilde{\pi}_{ \pm}-\mathrm{i} \frac{\kappa}{2} \tilde{v}_{ \pm}\right), \quad \tilde{a}_{ \pm}^{\dagger}=\frac{-\mathrm{i}}{\sqrt{2 \kappa}}\left(\tilde{\pi}_{\mp}+\mathrm{i} \frac{\kappa}{2} \tilde{v}_{\mp}\right)
$$

with nonzero commutators $\left[\tilde{a}_{ \pm}, \tilde{a}_{ \pm}^{\dagger}\right]=1$. Using the Fock representation for $(\tilde{v}, \tilde{\pi})$ and coordinate representation for $(\tilde{x}, \tilde{p})$, any state of this system is described by

$$
|\Psi(t)\rangle=\sum_{n_{+} \geq 0, n_{-} \geq 0} \int \mathrm{d} y\left|n_{+}, n_{-}\right\rangle \otimes|y\rangle \Phi_{n_{+} n_{-}}(y, t),
$$

where $\left|n_{+}, n_{-}\right\rangle$is the eigenstate of $\tilde{N}_{ \pm}=\tilde{a}_{ \pm}^{\dagger} \tilde{a}_{ \pm}$with eigenvalues $n_{ \pm} \in \mathbb{N} \cup\{0\}$ and $|y\rangle$ is the eigenstate of commuting operators $\tilde{x}_{i}$ with eigenvalue $y_{i}$. They are normalized as

$$
\left\langle n_{+}, n_{-} \mid n_{+}^{\prime}, n_{-}^{\prime}\right\rangle=\delta_{n_{+} n_{+}^{\prime}} \delta_{n_{-} n_{-}^{\prime}}, \quad\left\langle y \mid y^{\prime}\right\rangle=\delta^{2}\left(y-y^{\prime}\right) .
$$

The scalar product is given by

$$
\left\langle\Psi \mid \Psi^{\prime}\right\rangle=\sum_{n_{ \pm}} \int \mathrm{d} y \overline{\Phi_{n_{+} n_{-}}(y, t)} \Phi_{n_{+} n_{-}}^{\prime}(y, t) .
$$


In the quantization in the extended phase space the second class constraints (2.2) are imposed as physical state conditions by taking their non-hermitian combination,

$$
\tilde{a}_{ \pm}\left|\Psi_{\text {phys }}(t)\right\rangle=0 .
$$

This means that physical states are minimum uncertainty states in $(\tilde{v}, \tilde{\pi})$. Condition $(3.12)$ selects out only the $n_{+}=n_{-}=0$ state, so that $\Phi_{n_{+} n_{-}}(y, t)=0$ except for $\Phi_{0,0}(y, t) \equiv \Phi_{0}(y, t)$,

$$
\left|\Psi_{\text {phys }}(t)\right\rangle=\int \mathrm{d} y|0,0\rangle \otimes|y\rangle \Phi_{0}(y, t) .
$$

The Schrödinger equation is

$$
\left(\mathrm{i} \partial_{t}-H\right)\left|\Psi_{\text {phys }}(t)\right\rangle=0, \quad H=\frac{\hat{\tilde{p}}^{2}}{2 m},
$$

and thus

$$
\left(\mathrm{i} \partial_{t}-H\right) \Phi_{0}(y, t)=0, \quad H=\frac{1}{2 m}\left(-\mathrm{i} \partial_{y_{i}}\right)^{2} .
$$

The generators of the Weyl algebra are given in the extended space as polynomials $\mathfrak{G}(X, P)$ of the operator equivalent of (2.11), and, since they commute with $\tilde{a}_{ \pm}$and $\tilde{a}_{ \pm}^{\dagger}$, physical states remain physical ${ }^{6}$. They act on the physical states as

$$
\left.\Psi_{\text {phys }}(t)\right\rangle \quad \rightarrow \quad\left|\Psi_{\text {phys }}^{\prime}(t)\right\rangle=e^{\mathrm{i} \mathfrak{G}(X, P)}\left|\Psi_{\text {phys }}(t)\right\rangle
$$

and it turns out that the transformation of the wave function $\Phi_{0}(y, t)$ is

$$
\Phi_{0}^{\prime}(y, t)=e^{\mathrm{i} \mathfrak{G}(X, P)} \Phi_{0}(y, t)=e^{\mathrm{i} \mathfrak{G}\left(y-t\left(-\mathrm{i} \partial_{y}\right),\left(-\mathrm{i} \partial_{y}\right)\right)} \Phi_{0}(y, t) .
$$

This transformation has the same form as the one in the reduced phase space generated by (3.2)-(3.8). Then the wave function in the reduced space $\Psi(y, t)=\langle y \mid \Psi(t)\rangle$ and $\Phi_{0}(y, t)=$ $\langle y| \otimes\langle 00 \mid \Psi(t)\rangle$ that appear in the extended space quantization are identified. Note that in the former $\langle y|$ is eigenstate of $\hat{y}_{i}=x_{i}+\frac{\kappa}{2 m^{2}} \epsilon_{i j} p_{j}$ in (3.1) but $\langle y|$ in the latter is eigenstate of $\hat{\tilde{x}}_{i}$ that are commuting in the extended space.

We can see now how the non-commutativity of the position operators appears. $\hat{x}_{ \pm}=x_{1} \pm \mathrm{i} x_{2}$ are commuting in the extended phase space. Using (2.4) we write

$$
x_{+}=\tilde{x}_{+}+\mathrm{i} \frac{\kappa}{2 m^{2}} \tilde{p}_{+}+\mathrm{i} \sqrt{\frac{2 \kappa}{m^{2}}} \tilde{a}_{-}^{\dagger}, \quad x_{-}=\tilde{x}_{-}-\mathrm{i} \frac{\kappa}{2 m^{2}} \tilde{p}_{-}-\mathrm{i} \sqrt{\frac{2 \kappa}{m^{2}}} \tilde{a}_{-}=x_{+}^{\dagger} .
$$

In the reduced space quantization procedure, the $\tilde{a}_{ \pm}$are effectively put to zero and $x_{ \pm}$becomes a non-commutative operator on $|\Psi(t)\rangle$. On the other hand in the quantization in the extended space, expectation values of the position operators between two physical states are given by

$$
\begin{aligned}
\left\langle\Psi\left|\hat{x}_{ \pm}\right| \Psi^{\prime}\right\rangle & =\int \mathrm{d} y \mathrm{~d} y^{\prime} \overline{\Phi_{0}(y, t)}\left\langle y\left|\left\langle 0\left|\left(\tilde{x}_{ \pm} \pm \mathrm{i} \frac{\kappa}{2 m^{2}} \tilde{p}_{ \pm} \pm \mathrm{i} \sqrt{\frac{2 \kappa}{m^{2}}}\left(\begin{array}{l}
\tilde{a}_{-}^{\dagger} \\
\tilde{a}_{-}
\end{array}\right)\right)\right| 0\right\rangle\right| y^{\prime}\right\rangle \Phi_{0}^{\prime}\left(y^{\prime}, t\right) \\
& =\int \mathrm{d} y \overline{\Phi_{0}(y, t)}\left(y_{ \pm} \pm \mathrm{i} \frac{\kappa}{2 m^{2}}\left(-2 \mathrm{i} \partial_{y_{ \pm}}\right)\right) \Phi_{0}^{\prime}(y, t) .
\end{aligned}
$$

Commutative position operators $\hat{x}_{ \pm}$on states $|\Psi\rangle$ act as non-commutative operators $\left(y_{ \pm} \pm\right.$ $\left.\mathrm{i} \frac{\kappa}{2 m^{2}}\left(-2 \mathrm{i} \partial_{y_{ \pm}}\right)\right)$on the wave function $\Phi_{0}(y, t)$.

\footnotetext{
${ }^{6}$ The angular momentum $J$ in $(2.15)$ contains a term depending on $(v, \pi)$, but it commutes with $\tilde{a}_{ \pm}, \tilde{a}_{ \pm}^{\dagger}$.
} 
It is useful to consider the unitary transformation $U$ in (3.11) on the creation and annihilation operators $\tilde{a}_{ \pm}, \tilde{a}_{ \pm}^{\dagger}$,

$$
\tilde{a}_{+}=U^{\dagger} a_{+} U=a_{+}, \quad \tilde{a}_{-}=U^{\dagger} a_{-} U=a_{-}-\sqrt{\frac{\kappa}{2 m^{2}}} p_{-} .
$$

The quantization in the extended phase space can be also done by considering the constraint equations (3.12) in terms of the operators $a_{ \pm}, a_{ \pm}^{\dagger}$. The physical state conditions (3.12) are

$$
a_{+}\left|\Psi_{\text {phys }}(t)\right\rangle=0, \quad\left(p_{-}-\sqrt{\frac{2 m^{2}}{\kappa}} a_{-}\right)\left|\Psi_{\text {phys }}(t)\right\rangle=0
$$

and $\left|\Psi_{\text {phys }}\right\rangle$ is a coherent state of $a_{-}$with eigenvalue $\sqrt{\frac{\kappa}{2 m^{2}}} p_{-}[16]$. In this representation, the Schrödinger generators are

$$
\begin{aligned}
X_{ \pm}^{(2)}= & \left(x_{ \pm} \mp \mathrm{i} \frac{\kappa}{2 m^{2}} p_{ \pm}\right)-\frac{t}{m} p_{ \pm} \pm \mathrm{i} \frac{\kappa}{m^{2}}\left(p_{ \pm}-\sqrt{\frac{2 m^{2}}{\kappa}}\left(\begin{array}{c}
a_{-}^{\dagger} \\
a_{-}
\end{array}\right)\right) \\
P_{ \pm}^{(2)}= & p_{ \pm}=-2 \mathrm{i} \partial_{x_{\mp}}, \quad\left[x_{ \pm}, p_{\mp}\right]=2 \mathrm{i}, \\
D^{(2)}= & \frac{1}{2}\left(\left(x_{+} p_{-}+p_{+} x_{-}-\frac{2 t}{m} p_{+} p_{-}\right)+\mathrm{i} \frac{\kappa}{m^{2}}\left(p_{+}-\sqrt{\frac{2 m^{2}}{\kappa}} a_{-}^{\dagger}\right) p_{-}\right. \\
& \left.-\mathrm{i} \frac{\kappa}{m^{2}} p_{+}\left(p_{-}-\sqrt{\frac{2 m^{2}}{\kappa}} a_{-}\right)\right), \\
C^{(2)}= & \frac{1}{2}\left(\left(x_{+}-\mathrm{i} \frac{\kappa}{2 m^{2}} p_{+}\right)\left(x_{-}+\mathrm{i} \frac{\kappa}{2 m^{2}} p_{-}\right)\right. \\
& -\frac{t}{m}\left(\left(x_{+}-\mathrm{i} \frac{\kappa}{2 m^{2}} p_{+}\right) p_{-}+p_{+}\left(x_{-}+\mathrm{i} \frac{\kappa}{2 m^{2}} p_{-}\right)\right) \\
& +\frac{t^{2}}{2 m^{2}} p_{+} p_{-}+\frac{1}{2}\left(\left(x_{+}-\mathrm{i} \frac{\kappa}{2 m^{2}} p_{+}\right)-\frac{t}{m} p_{+}\right)\left(-\mathrm{i} \frac{\kappa}{m^{2}}\right)\left(p_{-}-\sqrt{\frac{2 m^{2}}{\kappa}} a_{-}\right) \\
& +\frac{1}{2} \mathrm{i} \frac{\kappa}{m^{2}}\left(p_{+}-\sqrt{\frac{2 m^{2}}{\kappa}} a_{-}^{\dagger}\right)\left(\left(x_{-}+\mathrm{i} \frac{\kappa}{2 m^{2}} p_{-}\right)-\frac{t}{m^{2}} p_{-}\right) \\
& \left.+\frac{1}{2}\left(\mathrm{i} \frac{\kappa}{m^{2}}\left(p_{+}-\sqrt{\frac{2 m^{2}}{\kappa}} a_{-}^{\dagger}\right)\right)\left(-\mathrm{i} \frac{\kappa}{m^{2}}\left(p_{-}-\sqrt{\frac{2 m^{2}}{\kappa}} a_{-}\right)\right)\right) . \\
J^{(2)}= & \frac{\mathrm{i}}{2}\left(\left(x_{+} p_{-}-p_{+} x+-\frac{\kappa}{m^{2}} p_{+} p_{-}\right)+\mathrm{i} \frac{\kappa}{m^{2}}\left(p_{+}-\sqrt{\frac{2 m^{2}}{\kappa}} a_{-}^{\dagger}\right) p_{-}\right. \\
+ & \left.\frac{\kappa}{m^{2}} p_{+}\left(p_{-}-\sqrt{\frac{2 m^{2}}{\kappa}} a_{-}\right)\right) .
\end{aligned}
$$

These generators commute with the constraint equations and with the Schrödinger operator $\mathrm{i} \partial_{t}-H$. Notice that the set of generators do not depend on $a_{+}, a_{+}^{\dagger}$, and therefore the transition to the Fock space used in [16] is recovered.

The Fock expression of a generic element of the Weyl algebra $\mathfrak{G}(X, P)$ can be obtained using the expression of the operators $X$ and $P$ given by (2.11). 


\subsubsection{Coordinate representation}

In the representation of coordinates the time Schrödinger equation and the constraint equations (3.13) in the non-commutative plane becomes [1]

$$
\begin{aligned}
& \hat{S}_{1} \Psi \equiv\left(\frac{\partial}{\partial v_{-}}+\frac{\kappa}{4} v_{+}\right) \Psi(x, v, t)=0, \\
& \hat{S}_{2} \Psi \equiv\left(\frac{\partial}{\partial x_{+}}-\mathrm{i} \frac{m}{4} v_{-}-\mathrm{i} \frac{m}{\kappa} \frac{\partial}{\partial v_{+}}\right) \Psi(x, v, t)=0, \\
& \hat{S}_{3} \Psi \equiv\left(\mathrm{i} \frac{\partial}{\partial t}+\frac{2}{m} \frac{\partial^{2}}{\partial x_{+} \partial x_{-}}\right) \Psi(x, v, t)=0 .
\end{aligned}
$$

In this representation, the operators associated to the generators of the Heisenberg algebra are

$$
\begin{aligned}
& \hat{P}_{1}=-\mathrm{i} \frac{\partial}{\partial x_{+}}-\mathrm{i} \frac{\partial}{\partial x_{-}}, \quad \hat{P}_{2}=\frac{\partial}{\partial x_{+}}-\frac{\partial}{\partial x_{-}}, \\
& \hat{\tilde{K}}_{1}=\frac{m}{2}\left(x_{+}+x_{-}\right)+\left(\mathrm{i} t-\frac{\kappa}{2 m}\right) \frac{\partial}{\partial x_{+}}+\left(\mathrm{i} t+\frac{\kappa}{2 m}\right) \frac{\partial}{\partial x_{-}}+\frac{\kappa}{4 \mathrm{i}}\left(v_{+}-v_{-}\right)+\mathrm{i} \frac{\partial}{\partial v_{+}}+\mathrm{i} \frac{\partial}{\partial v_{-}}, \\
& \hat{\tilde{K}}_{2}=\frac{m}{2 \mathrm{i}}\left(x_{+}-x_{-}\right)-\left(t+\mathrm{i} \frac{\kappa}{2 m}\right) \frac{\partial}{\partial x_{+}}+\left(t-\mathrm{i} \frac{\kappa}{2 m}\right) \frac{\partial}{\partial x_{-}}-\frac{\kappa}{4}\left(v_{+}+v_{-}\right)-\frac{\partial}{\partial v_{+}}+\frac{\partial}{\partial v_{-}},
\end{aligned}
$$

or, in covariant form,

$$
\hat{P}_{i}=-\mathrm{i} \frac{\partial}{\partial x_{i}}, \quad \hat{\tilde{K}}_{i}=m x_{i}+\mathrm{i} t \frac{\partial}{\partial x_{i}}+\mathrm{i} \frac{\kappa}{2 m} \epsilon_{i j} \frac{\partial}{\partial x_{j}}+\frac{\kappa}{2} \epsilon_{i j} v_{j}+\mathrm{i} \frac{\partial}{\partial v_{i}},
$$

which, indeed, satisfy $\left[\hat{P}_{i}, \hat{\tilde{K}}_{j}\right]=-\mathrm{i} m \delta_{i j}$, with all the other commutators equal to zero.

It is immediate to check that the operators $\hat{P}_{i}, \hat{\tilde{K}}_{i}$ commute with all of $\hat{S}_{1}, \hat{S}_{2}$ and $\hat{S}_{3}$, and hence that they generate Schrödinger symmetries for the free particle in the non-commutative plane. The rest of generators of the Schrödinger algebra are given by

$$
\begin{aligned}
\hat{H}= & -\frac{2}{m} \frac{\partial^{2}}{\partial x_{+} \partial x_{-}}=-\frac{1}{2 m} \frac{\partial^{2}}{\partial x_{i}{ }^{2}}, \\
\hat{J}= & -\mathrm{i} \epsilon_{i j} x_{i} \frac{\partial}{\partial x_{j}}+\frac{\kappa}{2 m^{2}} \frac{\partial^{2}}{\partial x_{i}{ }^{2}}-\mathrm{i} \frac{\kappa}{2 m} v_{i} \frac{\partial}{\partial x_{i}}-\frac{1}{m} \epsilon_{i j} \frac{\partial^{2}}{\partial x_{i} \partial v_{j}}, \\
\hat{D}= & -\mathrm{i} x_{i} \frac{\partial}{\partial x_{i}}+\frac{1}{m} t \frac{\partial^{2}}{\partial x_{i}{ }^{2}}+\frac{1}{m} \frac{\partial^{2}}{\partial x_{i} \partial v_{i}}+\mathrm{i} \frac{\kappa}{2 m} \epsilon_{i j} v_{i} \frac{\partial}{\partial x_{j}}-\mathrm{i}, \\
\hat{C}= & 2 \mathrm{i} t x_{i} \frac{\partial}{\partial x_{i}}+\mathrm{i} \frac{\kappa^{2}}{2 m^{2}} v_{i} \frac{\partial}{\partial x_{i}}+\mathrm{i} \frac{\kappa}{m} \epsilon_{i j} x_{i} \frac{\partial}{\partial x_{j}}-\mathrm{i} \frac{\kappa}{m} t \epsilon_{i j} v_{i} \frac{\partial}{\partial x_{j}} \\
& -\mathrm{i} \frac{\kappa}{m} \epsilon_{i j} v_{i} \frac{\partial}{\partial v_{j}}+2 \mathrm{i} x_{i} \frac{\partial}{\partial v_{i}}-\frac{1}{m} t^{2} \frac{\partial^{2}}{\partial x_{i}{ }^{2}}-\frac{\kappa^{2}}{4 m^{3}} \frac{\partial^{2}}{\partial x_{i}{ }^{2}}-\frac{1}{m} \frac{\partial^{2}}{\partial v_{i}{ }^{2}} \\
& -\frac{2}{m} t \frac{\partial^{2}}{\partial x_{i} \partial v_{i}}+\frac{\kappa}{m^{2}} \epsilon_{i j} \frac{\partial^{2}}{\partial x_{i} \partial v_{j}}+m x_{i}^{2}+\kappa \epsilon_{i j} x_{i} v_{j}+\frac{\kappa^{2}}{4 m} v_{i}^{2}+2 \mathrm{i} t .
\end{aligned}
$$

Using these expressions, one can check explicitly the commutators (3.9), and also that these quadratic generators commute with $\hat{S}_{1}, \hat{S}_{2}$ and $\hat{S}_{3}$ (this also follows from the derivation properties of the commutators and the corresponding commutation of the linear generators $\hat{P}_{i}, \hat{\tilde{K}}_{i}$, and this proves that the Schrödinger equation for the free particle in the noncommutative plane has the Schrödinger algebra as a symmetry. Notice, however, that in this coordinate representation of the non-reduced quantum space the quadratic operators contain second order derivatives, and 
hence do not generate point transformations for the coordinates $x, v$. This is in agreement with the results obtained in the reduced space quantization and the Fock space representation. In any case, the fact that the linear generators commute with $\hat{S}_{1}, \hat{S}_{2}$ and $\hat{S}_{3}$ allows to prove that the quadratic ones also commute, and thus generate symmetries of the Schrödinger equation of the free particle in the non-commutative plane.

\section{Acknowledgments}

We thank Jorge Zanelli for collaboration in some parts of this work and Mikhail Plyushchay for reading the manuscript. We also thank Adolfo Azcárraga and Jurek Lukierski for discussions, and Rabin Banerjee for letting us know about the results in [3]. CB was partially supported by Spanish Ministry of Economy and Competitiveness project DPI2011-25649. We also acknowledge partial financial support from projects FP2010-20807-C02-01, 2009SGR502 and CPAN Consolider CSD 2007-00042.

\section{References}

[1] Alvarez P.D., Gomis J., Kamimura K., Plyushchay M.S., (2+1)D exotic Newton-Hooke symmetry, duality and projective phase, Ann. Physics 322 (2007), 1556-1586, hep-th/0702014.

[2] Ballesteros A., Gadella M., del Olmo M.A., Moyal quantization of $(2+1)$-dimensional Galilean systems, J. Math. Phys. 33 (1992), 3379-3386.

[3] Banerjee R., Deformed Schrödinger symmetry on noncommutative space, Eur. Phys. J. C Part. Fields 47 (2006), 541-545, hep-th/0508224.

[4] Bekaert X., Meunier E., Moroz S., Symmetries and currents of the ideal and unitary Fermi gases, J. High Energy Phys. 2012 (2012), no. 2, 113, 59 pages, arXiv:1111.3656.

[5] Brihaye Y., Gonera C., Giller S., Kosiński P., Galilean invariance in $2+1$ dimensions, hep-th/9503046.

[6] Callan C.G., Coleman S.R., Wess J., Zumino B., Structure of phenomenological Lagrangians. II, Phys. Rev. 177 (1969), 2247-2250.

[7] Coleman S.R., Wess J., Zumino B., Structure of phenomenological Lagrangians. I, Phys. Rev. 177 (1969), 2239-2247.

[8] del Olmo M.A., Plyushchay M.S., Electric Chern-Simons term, enlarged exotic Galilei symmetry and noncommutative plane, Ann. Physics 321 (2006), 2830-2848, hep-th/0508020.

[9] Duval C., Horváthy P.A., Exotic Galilean symmetry in the non-commutative plane and the Hall effect, J. Phys. A: Math. Gen. 34 (2001), 10097-10107, hep-th/0106089.

[10] Eastwood M., Higher symmetries of the Laplacian, Ann. of Math. 161 (2005), 1645-1665, hep-th/0206233.

[11] Gomis J., Kamimura K., Schrödinger equations for higher order non-relativistic particles and $N$-Galilean conformal symmetry, Phys. Rev. D 85 (2012), 045023, 6 pages, arXiv:1109.3773.

[12] Hagen C.R., Scale and conformal transformations in Galilean-covariant field theory, Phys. Rev. D 5 (1972), 377-388.

[13] Hagen C.R., Galilean-invariant gauge theory, Phys. Rev. D 31 (1985), 848-855.

[14] Horváthy P.A., Martina L., Stichel P.C., Galilean symmetry in noncommutative field theory, Phys. Lett. B 564 (2003), 149-154, hep-th/0304215.

[15] Horváthy P.A., Martina L., Stichel P.C., Exotic Galilean symmetry and non-commutative mechanics, SIGMA 6 (2010), 060, 26 pages, arXiv:1002.4772.

[16] Horváthy P.A., Plyushchay M.S., Anyon wave equations and the noncommutative plane, Phys. Lett. B 595 (2004), 547-555, hep-th/0404137.

[17] Horváthy P.A., Plyushchay M.S., Nonrelativistic anyons in external electromagnetic field, Nuclear Phys. B 714 (2005), 269-291, hep-th/0502040.

[18] Ivanov E.A., Ogieveckii V.I., Inverse Higgs effect in nonlinear realizations, Theoret. and Math. Phys. 25 (1975), 1050-1059.

[19] Jackiw R., Introducing scale symmetry, Phys. Today 25 (1972), no. 1, 23-27. 
[20] Kastrup H.A., Gauge properties of the Galilei space, Nuclear Phys. B B7 (1968), 545-558.

[21] Lévy-Leblond J.M., Galilei group and Galilean invariance, in Group Theory and its Applications, Vol. II, Academic Press, New York, 1971, 221-299.

[22] Lukierski J., Stichel P.C., Zakrzewski W.J., Galilean-invariant (2+1)-dimensional models with a ChernSimons-like term and $D=2$ noncommutative geometry, Ann. Physics 260 (1997), 224-249, hep-th/9612017.

[23] Niederer U., The maximal kinematical invariance group of the free Schrödinger equation, Helv. Phys. Acta 45 (1972), 802-810.

[24] Valenzuela M., Higher-spin symmetries of the free Schrödinger equation, arXiv:0912.0789.

[25] Vasiliev M.A., Higher spin gauge theories in any dimension, C. R. Phys. 5 (2004), 1101-1109, hep-th/0409260. 